\title{
Simulating solar MHD
}

\author{
M. Schüssler \\ Kiepenheuer-Institut für Sonnenphysik (KIS) Schöneckstr. 6, D-79104 Freiburg, Germany \\ e-mail: msch@kis.uni-freiburg.de
}

Received: 5 October 1998 / Accepted: 29 October 1998

\begin{abstract}
Two aspects of solar MHD are discussed in relation to the work of the MHD simulation group at KIS. Photospheric magneto-convection, the nonlinear interaction of magnetic field and convection in a strongly stratified, radiating fluid, is a key process of general astrophysical relevance. Comprehensive numerical simulations including radiative transfer have significantly improved our understanding of the processes and have become an important tool for the interpretation of observational data. Examples of field intensification in the solar photosphere ('convective collapse') are shown. The second line of research is concerned with the dynamics of flux tubes in the convection zone, which has far-reaching implications for our understanding of the solar dynamo. Simulations indicate that the field strength in the region where the flux is stored before erupting to form sunspot groups is of the order of $10^{5} \mathrm{G}$, an order of magnitude larger than previous estimates based on equipartition with the kinetic energy of convective flows.
\end{abstract}

Key words. Solar physics, astrophysics and astronomy (photosphere and chromosphere; stellar interiors and dynamo theory; numerical simulation studies).

\section{Introduction}

The interaction between flows and magnetic field in the lower atmosphere (photosphere) and the convective envelope of the sun gives rise to a host of processes that are of general astrophysical interest: generation of magnetic flux in a self-excited hydromagnetic dynamo process near the base of the convection zone, the transport of flux in the form of magnetic flux tubes through the convective envelope, its emergence at the photosphere, the formation of sunspots and other magnetic structures, their interaction with the photospheric velocity fields channeling mechanical energy to heat the upper atmosphere and to drive the solar wind.

Due to the nonlinear character and complicated nature of the processes, comprehensive numerical simulations have become an increasingly important tool. Simulations performed by the MHD group at the Kiepenheuer-Institute for Solar Physics (KIS) and its collaborators in various places have concentrated on two areas of research: (1) radiative magneto-convection in the solar (sub-)photosphere, and (2) the dynamics of magnetic flux tubes in the convection zone, whose emergence at the surface gives rise to sunspot groups. In both lines of research, the reciprocation of simulation results and observational data has played a key role. The observable consequences of the simulations are compared with measurements, allowing us to calibrate simulation parameters or initial values. Information about processes or conditions not accessible to direct observation can this be inferred.

\section{MHD simulation at KIS}

\subsection{Photospheric magneto-convection}

The solar atmosphere represents a superb laboratory to study the physical processes arising from the interaction between convective flows, magnetic fields, and radiation in a strongly stratified fluid. The richness of structure in the various layers of the solar envelope, from sunspots to coronal loops, owes its existence to this intricate interplay and basic processes like coronal heating and wind acceleration are tightly connected to magnetoconvection. The large values of the (hydrodynamic and magnetic) Reynolds numbers and the strong stratification of the solar atmosphere lead to a wide spectrum of structure sizes with the photospheric scale height of about $100 \mathrm{~km}$ playing a prominent part. As a consequence, the sun is the only astrophysical object that allows us, in principle, to observe magneto-convection 
on its natural spatial and temporal scales. Even though, all observation (imaging, spectroscopy, polarimetry) involves integration over the line of sight, so that the height dimension appears in the data only in a convoluted form. Furthermore, the layers below optical depth unity (the solar 'surface') are inaccesible to direct observation although an important part of the magnetoconvective interaction takes place in the region of large superadiabaticity of the stratification down to $\simeq 1000 \mathrm{~km}$ below the surface. Comprehensive numerical simulations are performed in order to bridge the gap between the physical processes and the observational information: the simulations allow us to study the processes under consideration without restrictions on visibility and, at the same time, provide diagnostic information (synthetic images, line profiles and Stokes parameters) which can be compared with the measurements and so enable us to interpret the observations on the basis of comprehensive and consistent physical models.

The activities of the MHD simulation group at KIS (whose variable membership and cooperations can be inferred from the author lists of the publications cited below) began in the early 1980s, then at the UniversitätsSternwarte Göttingen. We started with simulations of stationary small-scale magnetic flux concentrations in the (sub)photosphere using a 2D-Moving-Finite-Element (MFE) code and incorporating radiative energy transfer by way of the diffusion approximation (Deinzer et al., 1984a, b). Extended parameter studies and the simulation of larger magnetic structures followed (Knölker et al., 1988; Knölker and Schüssler, 1988). A major step forward was the inclusion of a realistic model for the radiative transfer (integration of the transfer equation along a large number of rays) and the development of a comprehensive diagnostic package to compute synthetic continuum radiation maps and the profiles of spectral lines in unpolarized and polarized light (Stokes parameters), including the corresponding contribution functions (Grossmann-Doerth et al., 1989; Knölker et al., 1991; Schüssler, 1990, 1991). The progress made with the MFE code concerning solar magnetic elements in summarized in Grossmann-Doerth et al. (1994). In the meantime, we had developed a new 2D-code (Steiner et al., 1994), which permits a much larger spatial resolution through application of adaptive mesh refinement. With this code we are able to simulate full dynamics of solar surface convection and its interaction with magnetic field and radiation (Steiner et al., 1996, 1998). We are now in the process of extending this code to $3 \mathrm{D}$.

Recent results of our simulations concern the concentration of photospheric magnetic flux into isolated intense flux elements and the intensification of their field strength up to the observed kilogauss values (Solanki, 1993). 'Flux expulsion' in cellular convective flows leads to the observed concentration of the magnetic flux at the edges (downflow lanes) of granular and supergranular cells. Under solar conditions, however, this kinematical effect alone is not able to increase the field strength significantly above the equipartition value of about $500 \mathrm{G}$. The Lorentz force suppresses further growth of the field by impeding the horizontal flow. Further amplification of the magnetic field strength is due to thermal effects. Since the horizontal flows of granular convection carry heat to the downflow regions, the retardation of the flows by the growing magnetic field leads to an enhanced cooling of the magnetic region because the radiative losses cannot be balanced by the reduced horizontal flow. The cooling and the strongly superadiabatic stratification of the uppermost layers of the convection zone accelerate the downflow along the field lines (Parker, 1978; Spruit and Zweibel, 1979) and, since the horizontal flow is suppressed by the Lorentz force, a partial evacuation of the upper layers ensues. Pressure equilibrium with the surrounding gas is restored by compression of the flux tube, increasing the magnetic pressure. In this way, the magnetic field can be locally intensified to values which are only limited by the confining pressure of the external gas.

We have carried out numerical experiments in order to study these processes in detail and to determine their observable consequences (GrossmannDoerth et al., 1998). We began with the simulation of a field-free plasma, starting with a plane-parallel stratification taken from a model of the solar envelope. We then perturbed this convectively unstable configuration by a velocity field of small amplitude and let the system evolve until it relaxed to a 'normal' pattern of instationary convective motion. Next we imposed a homogeneous, vertical magnetic field onto the entire computational domain and followed the ensuing interaction between the magnetic field and the convective motions. Figure 1 shows stills from two simulation runs: the panels on the left-hand side give the field line patterns at various instants for an initial magnetic field of $100 \mathrm{G}$. The right-hand column corresponds to an initial field of $200 \mathrm{G}$, the second simulation starting from a different convection pattern than the first.

Two features of these results are most relevant with respect to observations. Firstly, strong vertical magnetic flux concentrations with $\mathrm{kG}$ fields are formed in regions of convective downflow. This effect is due to flux expulsion by the horizontal flows and convective collapse. Secondly, magnetic flux that is not swept to the vertical flux concentrations is wrapped up by the convective pattern and brought to the surface by upflows, where it forms patches of horizontal field with lower field strength, corresponding roughly to equipartition between magnetic and kinetic energy density. Both features, strong vertical flux concentrations and weak horizontal fields, are prominent in the observational results (e.g., Lites et al., 1998). As can be seen in Fig. 1, the photospheric size of the magnetic features with $\mathrm{kG}$ field strength is of the order of $100 \mathrm{~km}$. Furthermore, the occasional development of strong upflows with shocks is found whenever the downflow connected with the convective collapse becomes sufficiently vigorous that it suffers a 'rebound' in the deeper layers, where the density 

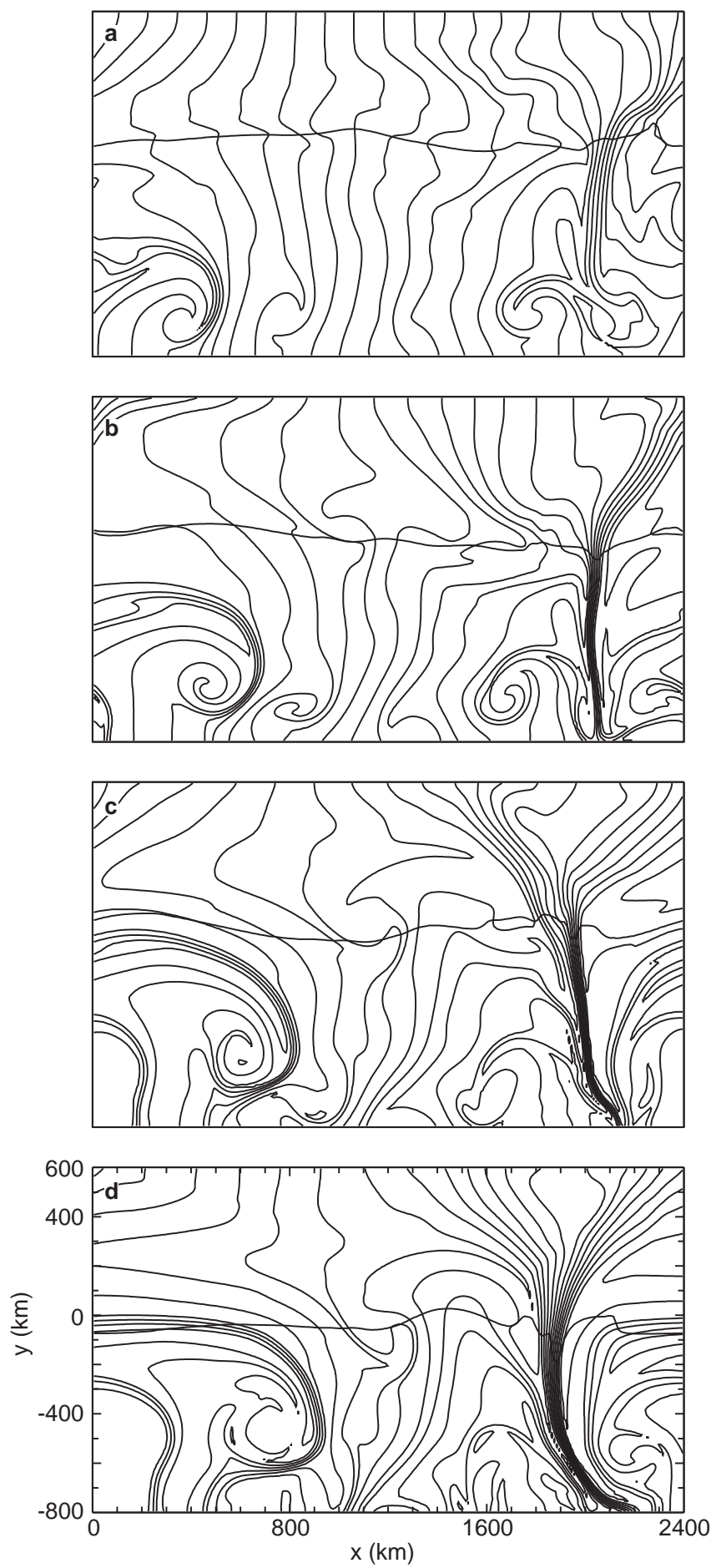

Fig. 1. Strong vertical and weaker horizontal magnetic field arising in a MHD simulation under conditions prevailing in the solar photosphere. The panels on the left-hand side show magnetic field lines at the times $60 \mathrm{~s}, 115 \mathrm{~s}, 175 \mathrm{~s}$, and $230 \mathrm{~s}$ after imposing an initially homogeneous magnetic field of $100 \mathrm{G}$. The panels on the right-hand side correspond to $100 \mathrm{~s}, 200 \mathrm{~s}, 250 \mathrm{~s}$, and $300 \mathrm{~s}$ and an initial field of $200 \mathrm{G}$, starting from a different convection pattern. The direction of the $x$-coordinate is parallel to the solar surface, spanning

increases rapidly. These upflows are possibly related to the spicule phenomenon. Other dynamical processes resulting from the interaction between strong magnetic flux concentrations and convective flow are transversal
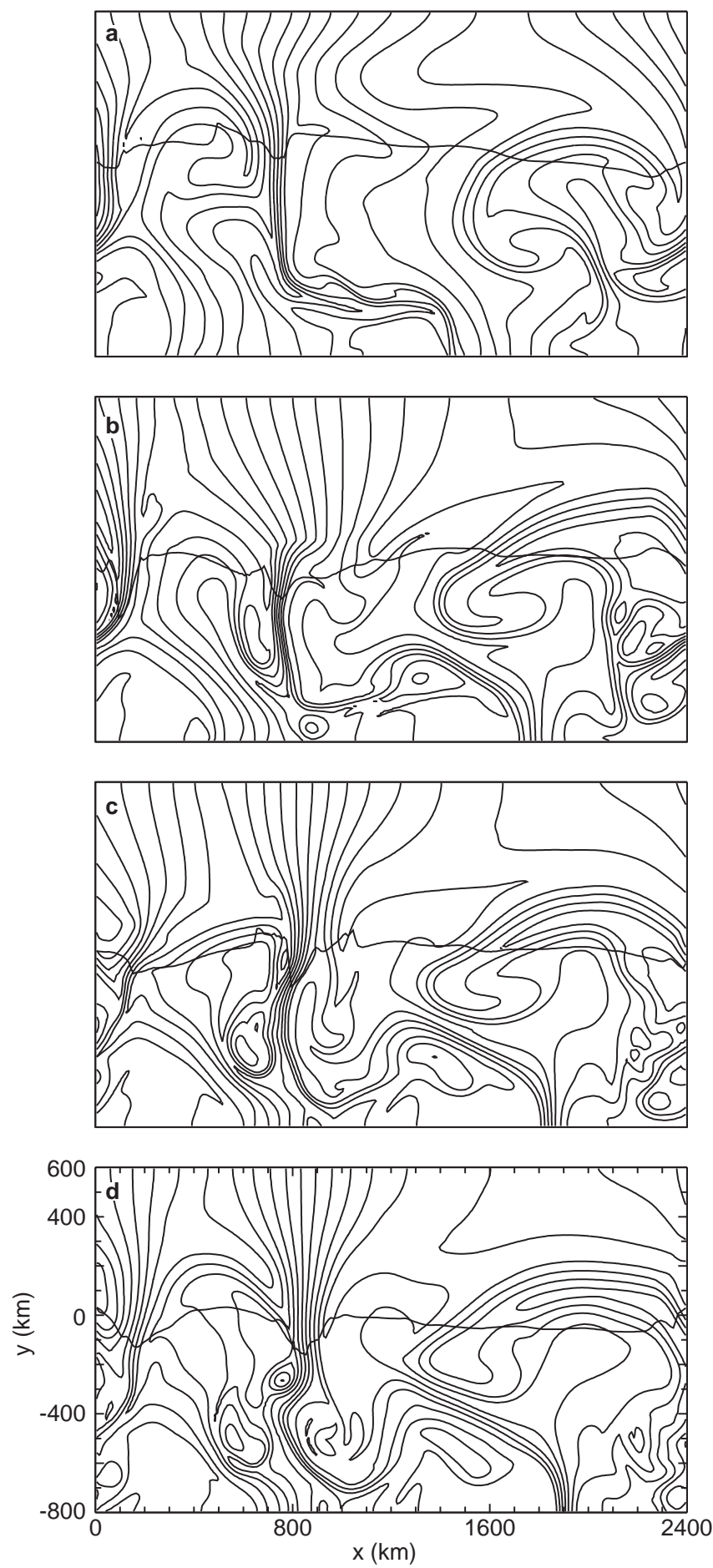

a distance of $2400 \mathrm{~km}$. The $y$-coordinate runs $1400 \mathrm{~km}$ in the vertical (height) direction, ranging from $800 \mathrm{~km}$ below the surface (optical depth unity at $500 \mathrm{~nm}$ for a mean solar atmosphere model) to $600 \mathrm{~km}$ above it. Top and bottom boundaries are open for the flow; the side boundaries are periodic. The horizontally running dotted curve indicates optical depth unity for the continuum radiation at $500 \mathrm{~nm}$ wavelength

oscillations and shock excitation (Steiner et al., 1998), which may contribute to the heating of higher atmospheric layers. 


\subsection{Flux-tube dynamics in the convection zone}

The observed properties of sunspot groups on the surface of the sun are consistent with the concept of magnetic flux tubes emerging from deep within the solar convection zone. In order to maintain coherence and orientation during their rise in the turbulent convective flows, the magnetic field strength in these flux tubes should always exceed the equipartition value with respect to the kinetic energy density of the convective motions (Moreno-Insertis, 1992).

Through linear stability analysis (Ferriz-Mas and Schüssler, 1993, 1995) and numerical simulations of rising flux tubes (Caligari et al., 1995, 1998) a consistent picture of the storage, instability, and rise of magnetic flux tubes has emerged (Schüssler et al., 1994; Schüssler 1996a), which is in accordance with the observed basic properties of sunspot groups: magnetic flux tubes are generated and stored in mechanical equilibrium in a subadiabatically stratified overshoot layer below the convection zone; they become unstable with respect to an undulatory (Parker-type) instability once the field strength exceeds a critical value; flux loops form, move through the convection zone and give rise to bipolar sunspot groups when they emerge at the surface. Both the stability criteria and the constraints set by the observed properties of sunspot groups (orientation, tilt angle, asymmetry, proper motions) require that the field strength at the bottom of the convection zone should be of the order of $10^{5} \mathrm{G}$, an order of magnitude larger than the equipartition field strength with respect to the convective flows.

Figures 2 and 3 show an example of the simulation results. The two transparent half-spheres in Fig. 2 correspond to the solar surface and the bottom of the convection zone, respectively. The observer looks faceon onto the erupting part of the flux tube, which can be recognized by its expansion. The tilt with respect to the

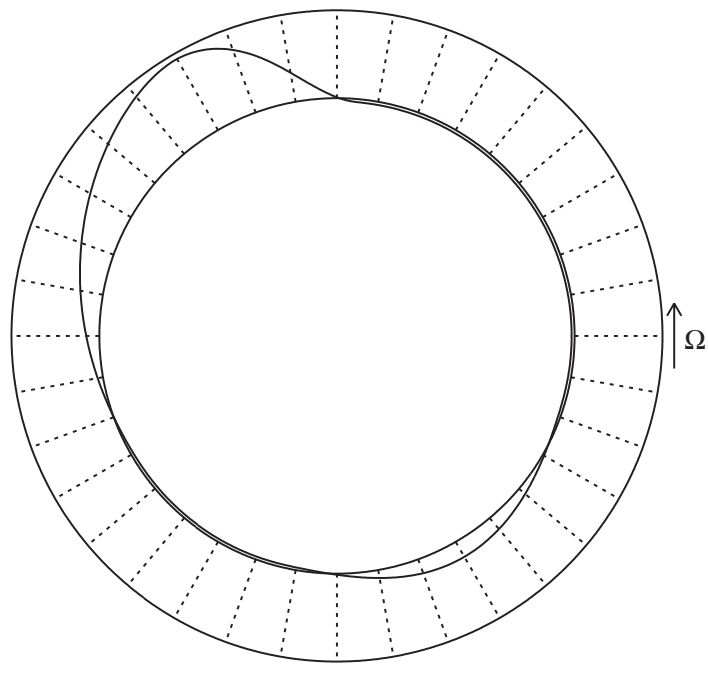

Fig. 3. Polar view corresponding to the flux tube configuration of Fig. 2. Shown is a projection of the tube on the equatorial plane. Note the distinct geometric asymmetry between the two legs of the emerging loop. Since the unstable mode has an azimuthal wave number $m=2$, a second loop can be seen emerging at about $180^{\circ}$ longitude distance from the first

direction of rotation is clearly visible. A slight poleward deflection of the erupted loop is visible, the effect being moderate since the initial field strength is large enough for buoyancy to dominate over the Coriolis force. Figure 3 gives a polar view on the same tube, i.e., a projection on the equatorial plane seen from above the north pole. An asymmetry between the preceding and the following part of the loop (with respect to the direction of rotation) is clearly visible: the following part is more vertical than the preceding part. The rise of such an asymmetric structure leads to the characteristic proper motions and geometrical asymmetries of young sunspot groups.

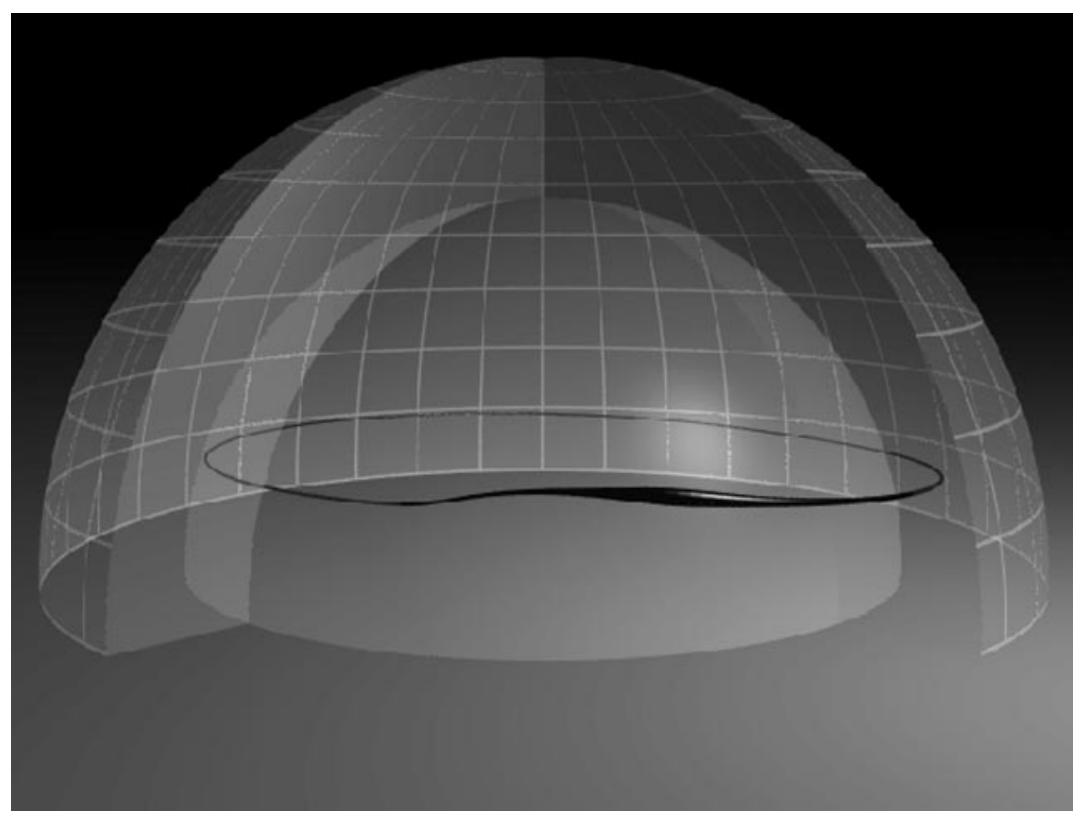

Fig. 2. Three-dimensional view of an emerging flux loop resulting from the undulatory instability. The northern hemisphere is represented. The two transparent half-spheres correspond to the solar surface and the bottom of the convection zone, respectively 
In summary, we may say that the combined approach of linear stability analysis and numerical simulation leads to a consistent model for the storage, instability and eruption of magnetic flux, which is in accordance with the observational results for sunspot groups and indicates the existence of a layer of strong, super-equipartition field in a stably stratified overshoot layer below the main body of the solar convection zone.

\section{Outlook}

In the field of photospheric magneto-convection, we are in the process of developing and testing a state-of-theart 3D-MHD-code with a Riemann solver on an unstructured, adaptive grid. The work on flux-tube dynamics is being extended to stars other than the sun, namely those rapid rotators showing prominent spots near their rotational poles (Schüssler 1996b; Schüssler et al., 1996).

\section{References}

Caligari, P., F. Moreno-Insertis, and M. Schüssler, Emerging flux tubes in the solar convection zone. I Asymmetry, tilt, and emergence latitude, Astrophys. J., 441, 886-902, 1995.

Caligari, P., F. Moreno-Insertis, and M. Schüssler, Emerging flux tubes in the solar convection zone. II The influence of initial conditions, Astrophys. J., 502, 481-492, 1998.

Deinzer, W., G. Hensler, M. Schüssler, and E. Weisshaar, Model calculations of magnetic flux tubes. I Equations and method, Astron. Astrophys., 139, 426-434, 1984a.

Deinzer, W., G. Hensler, M. Schüssler, and E. Weisshaar, Model calculations of magnetic flux tubes. II Stationary results for solar magnetic elements, Astron. Astrophys., 139, 435-449, 1984b.

Ferriz Mas, A., and M. Schüssler, Instabilities of magnetic flux tubes in a stellar convection zone. I Equatorial flux rings in differentially rotating stars, Geophys. Astrophys. Fluid Dyn., $\mathbf{7 2}$, 209-247, 1993.

Ferriz Mas, A., and M. Schüssler, Instabilities of magnetic flux tubes in a stellar convection zone. II Flux rings outside the equatorial plane Geophys. Astrophys. Fluid Dyn., 81, 233-265, 1995.

Grossmann-Doerth, U., M. Knölker, M. Schüssler, and E. Weisshaar, Models of magnetic flux sheets, in R.J. Rutten, G. Severino (eds.), Solar and Stellar Granulation, NATO/ARW, Kluwer, Dordrecht, pp. 481- 492, 1989.

Grossmann-Doerth, U., M. Knölker, M. Schüssler, and S. K. Solanki, The deep layers of solar magnetic elements, Astron. Astrophys., 285, 648-654, 1994.
Grossmann-Doerth, U., M. Schüssler, and O. Steiner, Convective intensification of solar surface magnetic fields: results of numerical experiments, Astron. Astrophys., 337, 928-939, 1998.

Knölker, M., and M. Schüssler, Model calculations of magnetic flux tubes. IV Convective energy transport and the nature of intermediate size flux concentrations, Astron. Astrophys., 202, 275-283, 1988.

Knölker, M., M. Schüssler, and E. Weisshaar, Model calculations of magnetic flux tubes. III Properties of solar magnetic elements, Astron. Astrophys.,. 194, 257-267, 1988.

Knölker, M., U. Grossmann-Doerth, M. Schüssler, and E. Weisshaar, Some developments in the theory of magnetic flux concentrations in the solar atmosphere, Adv. Space Res., 11, 285-295, 1991.

Lites, B. W., A. Skumanich, and V. Martínez Pillet, Vector magnetic fields of emerging solar flux. I Properties at the site of emergence, Astron. Astrophys., 333, 1053-1068, 1998.

Moreno-Insertis, F., The motion of magnetic flux tubes in the convection zone and the subsurface origin of active regions, in J. H. Thomas, N.O. Weiss (eds.), Sunspots-theory and observations, Kluwer, Dordrecht, 385-410, 1992.

Parker, E. N., Hydraulic concentration of magnetic fields in the solar photosphere. VI Adiabatic cooling and concentration in downdrafts, Astrophys. J., 221, 368-377, 1978.

Schüssler, M., Theoretical aspects of small-scale photospheric magnetic fields, in J.O. Stenflo (ed.), solar photosphere: structure, convection and magnetic fields, IAU-Symposium No. 138, Kluwer, Dordrecht, pp.161-179, 1990.

Schüssler, M., Solar magnetic elements, Geophys. Astrophys. Fluid Dyn., 62, 271-281, 1991.

Schüssler, M., Magnetic flux tubes and the solar dynamo, in K. Tsinganos (ed.), Solar and astrophysical magnetohydrodynamic flows, Kluwer, Dordrecht, pp. 17-37, 1996a.

Schüssler, M., Distribution of starspots on cool stars, in K. Strassmeier (ed.), Stellar surface structure, IAU-Symp. No. 176, Kluwer, Dordrecht, pp. 269-287, 1996b.

Schüssler, M., P. Caligari, A. Ferriz Mas, and F. Moreno-Insertis, Instability and eruption of magnetic flux tubes in the solar convection zone, Astron. Astrophys., 281, L69-L72, 1994.

Schüssler, M., P. Caligari, A. Ferriz-Mas, S. K. Solanki, and M. Stix, Distribution of starspots on cool stars. I Young and main sequence stars of $1 M_{\odot}$, Astron. Astrophys., 314, 503-513, 1996.

Solanki, S. K., Small-scale solar magnetic fields: an overview, Space Sci. Rev., 63, 1-188, 1993.

Spruit, H. C., and E. G. Zweibel, Convective instability of thin flux tubes, Solar Phys., 62, 15-22, 1979.

Steiner, O., M. Knölker, and M. Schüssler, Dynamic interaction of convection with magnetic flux sheets: First results of a new MHD code, in R. Rutten, C. Schrijver (eds.), Solar surface magnetism, NATO ARW, Kluwer, Dordrecht, pp. 441-470, 1994.

Steiner, O., U. Grossmann-Doerth, M. Knölker, and M. Schüssler, Polarized radiation diagnostics of magnetohydrodynamic models of the solar atmosphere, Solar Phys., 164, 223-242, 1996.

Steiner, O., U. Grossmann-Doerth, M. Knölker, and M. Schüssler, Dynamical interaction of solar magnetic elements and granular convection: results of a numerical simulation, Astrophys. J., 495, 468-484, 1998. 